\title{
IBS in a CAM-Dominated Electron Beam
}

\author{
A. Burov ${ }^{*}$, I. Gusachenko ${ }^{\S}$, S. Nagaitsev ${ }^{*}$ and A. Shemyakin ${ }^{*}$ \\ *Fermi National Accelerator Laboratory, P.O. Box 500, Batavia IL 60543 \\ ${ }^{\S}$ Novosibirsk State University, Novosibirsk, 630090, Russia
}

\begin{abstract}
Electron cooling of the $8.9 \mathrm{GeV} / \mathrm{c}$ antiprotons in the Recycler ring requires highquality dc electron beam with the current of several hundred $\mathrm{mA}$ and the kinetic energy of 4.3 $\mathrm{MeV}$. That high electron current is attained through beam recirculation (charge recovery). The primary current path is from the magnetized cathode at high voltage terminal to the ground, where the electron beam interacts with the antiproton beam and cooling takes place, and then to the collector in the terminal The energy distribution function of the electron beam at the collector determines the required collector energy acceptance. Multiple and single intra-beam scattering as well as the dissipation of density micro-fluctuations during the beam transport are studied as factors forming a core and tails of the electron energy distribution. For parameters of the Fermilab electron cooler, the single intra-beam scattering (Touschek effect) is found to be of the most importance.
\end{abstract}

Keywords : Touschek effect, coupled optics, canonical angular momentum.

PACS: 29.27.Bd, 29.27.Eg, 29.27.Fh

\section{INTRODUCTION}

In the Fermilab e-cooler ${ }^{1}$, energy distribution of the electrons at the collector affects the current loss and, consequently, possibility of operations in the DC mode. Core of the energy distribution is formed by multiple intra-beam scattering (IBS), as well as the dissipation of density micro-fluctuations. Extended tails of the electron energy distribution, significant for the charge recovery, are formed by single IBS, or Touschek effect. Because the electron beam is CAM-dominated ${ }^{2}$; conventional IBS results (as Bjorken-Mtingwa, Piwinski-Martini) cannot be applied. Both multiple and single IBS phenomena are treated here on a base of the Landau collision integral; the present paper is a more extended and refined version of Ref. ${ }^{4}$.

\begin{tabular}{|l|c|c|c|}
\hline TABLE 1. Fermilab e-cooler parameters. & Symbol & Value & Units \\
\hline Parameter & $I_{e}$ & $0.1-0.5$ & $\mathrm{~A}$ \\
\hline Beam current & $p$ & 4.8 & $\mathrm{MeV} / \mathrm{c}$ \\
\hline Electron momentum & $r_{c}$ & 0.38 & $\mathrm{~cm}$ \\
\hline Cathode radius & $T_{c}$ & 0.11 & $\mathrm{eV}$ \\
\hline Electron temperature at the cathode & $l$ & 99 & $\mathrm{~m}$ \\
\hline Length of trajectory & $U_{\text {coll }}$ & $2-4$ & $\mathrm{kV}$ \\
\hline Collector potential with respect to the cathode & $B_{c}$ & 90 & $\mathrm{G}$ \\
\hline Longitudinal magnetic field on the cathode & &
\end{tabular}




\section{CORE OF THE DISTRIBUTION}

An electron with a longitudinal velocity $v_{\|}<<c$ in the beam frame has the energy deviation $U=p v_{\|}=\gamma \beta m c v_{\|}$in the laboratory frame. This value is not changed by acceleration and deceleration; thus, according to kinematics, the beam longitudinal temperature $T_{\|} \equiv m \overline{v_{\|}^{2}} \cong m T_{c}^{2} / p^{2}$ gets to be extremely small with the acceleration.

IBS transfers high transverse temperature of electrons, which is not changed by the acceleration, to low longitudinal temperature; the evolution of the longitudinal distribution is described by the Landau kinetic equation ${ }^{3}$. When $T_{\|}<<T_{\perp}$, the kinetic equation on the longitudinal distribution function $f_{\|}\left(v_{\|}\right)$reduces to a pure diffusion with the diffusion coefficient independent on the longitudinal velocity:

$$
\frac{\partial f_{\|}}{\partial t}=\frac{D}{2} \frac{\partial^{2} f_{\|}}{\partial v_{\|}^{2}}, \quad D=4 \pi n_{e} r_{e}^{2} c^{4} L_{C} \iint d^{2} \mathbf{v} d^{2} \mathbf{v}^{\prime} \frac{f_{\perp}(\mathbf{v}) f_{\perp}\left(\mathbf{v}^{\prime}\right)}{\left|\mathbf{v}-\mathbf{v}^{\prime}\right|}
$$

Here $\mathbf{v}$ is $2 \mathrm{D}$ transverse velocity vector, and the electron density $n_{e}$ is supposed to be constant (Pierce regime) over the elliptic cross-section with half-axes $a_{\xi}, a_{\eta}$. Assuming the transverse distribution $f_{\perp}(\mathbf{v})$ to be Gaussian with main axes r. m. s. velocities $\overline{v_{\tilde{x}}^{2}}, \overline{v_{\tilde{y}}^{2}}$, it yields

$$
D=\frac{2 \pi^{3 / 2} n_{e} r_{e}^{2} c^{4} L_{C}}{\sqrt{\overline{v_{\tilde{x}}^{2}} \overline{v_{\tilde{y}}^{2}}}} \int_{0}^{2 \pi} \frac{d \varphi}{2 \pi} \frac{1}{\sqrt{\frac{\cos ^{2} \varphi}{\overline{v_{\tilde{x}}^{2}}}+\frac{\sin ^{2} \varphi}{\overline{v_{\tilde{y}}^{2}}}}}
$$

At the end of the transfer line, beam acquires energy spread given by an integral over the beam line:

$$
\overline{U_{I B S}^{2}}=\frac{2 \sqrt{\pi} I_{e} r_{e}^{2} L_{C}}{e c^{2} \varepsilon_{4 n}}\left(m c^{2}\right)^{2} \int_{0}^{l} d z \int_{0}^{2 \pi} \frac{d \varphi}{2 \pi} \frac{1}{\sqrt{\frac{\cos ^{2} \varphi}{\overline{v_{\tilde{x}}^{2}}}+\frac{\sin ^{2} \varphi}{\overline{v_{\tilde{y}}^{2}}}}},
$$

where $\varepsilon_{4 n}=a_{\xi} a_{\eta} \sqrt{\overline{v_{\tilde{x}}^{2}} \overline{v_{\tilde{y}}^{2}}}$ is a normalized 4D emittance, $L_{c}=\ln \left(r_{\max } / r_{\min }\right)$ is the Coulomb logarithm. For the design optics ${ }^{5}$, this yields $\sqrt{\overline{U_{I B S}^{2}}} \approx 75 \mathrm{eV}$.

Another source of longitudinal temperature growth is dissipation of density fluctuations in the beam. Excessive potential energy (beam frame) $U_{e x c} 2 e^{2} n^{1 / 3}$ (see Ref $^{6}$ ) transforms into the r. m. s. energy spread

$$
\overline{U_{D F}^{2}} \cong 2(\gamma \beta)^{5 / 3} r_{e}\left(\frac{I_{e}}{\pi e c a_{e}^{2}}\right)^{1 / 3}\left(m c^{2}\right)^{2}
$$


For $I_{e}=0.2 \mathrm{~A}$, this yields $\sqrt{\overline{U_{D F}^{2}}} \cong 50 \mathrm{eV}$, and $\sqrt{\overline{U^{2}}}=\sqrt{\overline{U_{I B S}^{2}}+\overline{U_{D F}^{2}}}=90 \mathrm{eV}$.

\section{TAILS OF THE DISTRIB UTION}

A portion of particles $\Delta(U)$ which energy deviation exceeds a given collector potential $U$ is referred here as losses. Losses as low as ? $(U)=10^{-6}$ correspond to $U=4.75 \sqrt{\overline{U^{2}}}=430 \mathrm{~V}$ low-energy tail of the Gaussian distribution $f(U)$. However, when the loss level is so small, it is determined rather by single scatterings (Touschek), than by multiple ones. In non-relativistic case, the Rutherford differential cross-section gives a cross-section for events when one of the scattering particles acquires longitudinal velocity larger than $v_{\|}$, assuming $v_{0}$ as a relative velocity:

$$
\sigma\left(v_{0}, v_{\|}\right)=\int_{0}^{2 \pi} d \varphi \cdot 2 \int_{0}^{\arccos \left(2 v_{\|} / v_{0}\right)} \frac{d \sigma}{d o} \sin \theta d \theta=\frac{4 \pi r_{e}^{2} c^{4}}{v_{0}^{2} v_{\|}^{2}}\left(1-\frac{4 v_{\|}^{2}}{v_{0}^{2}}\right)
$$

The instantaneous loss rate in the beam frame is

$$
\frac{1}{n_{e}} \frac{d n_{e}}{d t}=\frac{n_{e}}{2} \int d \mathbf{v} d \mathbf{v}^{\prime} \sigma\left(\left|\mathbf{v}-\mathbf{v}^{\prime}\right|, v_{\|}\right)\left|\mathbf{v}-\mathbf{v}^{\prime}\right| f_{\perp}(\mathbf{v}) f_{\perp}\left(\mathbf{v}^{\prime}\right) .
$$

For the Gaussian transverse distribution, the above 4D integral reduces to a single integral

$$
\begin{gathered}
\Delta(U)=\frac{\sqrt{\pi} I_{e} r_{e}^{2} L_{C}}{e c^{2} \varepsilon_{4 n}}\left(\frac{m c^{2}}{U}\right)^{2} \int_{0}^{l} d z \int_{0}^{2 \pi} \frac{d \varphi}{2 \pi} \frac{\mathrm{S}(\Xi(\varphi))}{\sqrt{\frac{\cos ^{2} \varphi}{v_{\tilde{x}}^{2}}+\frac{\sin ^{2} \varphi}{\overline{v_{\tilde{y}}^{2}}}}} \\
\Xi(\phi)=\frac{U}{p} \sqrt{\frac{\cos ^{2} \phi}{\overline{v_{\tilde{x}}^{2}}}+\frac{\sin ^{2} \phi}{v_{\tilde{y}}^{2}}}, \quad \mathrm{~S}(x)=\left(1+2 x^{2}\right) \operatorname{erfc}(x)-\frac{2 x}{\sqrt{\pi}} \exp \left(-x^{2}\right) .
\end{gathered}
$$

For the $99 \mathrm{~m}$ long cooling line, the losses vary moderately $(<50 \%)$ for drastically different beam envelopes. For the short U-bend line ${ }^{7}$, the calculated dependence on the envelope is much stronger. Increase of the beam size leads to significant drop of the losses. Fig. 1 shows calculated (line) and measured (dots) dependences of the beam losses versus the collector potential for design optics of the cooling line (left) and the U-bend line (right). For the cooling line, the gowing discrepancy at high voltage is thought to be caused by secondary electrons escaped from the collector. 

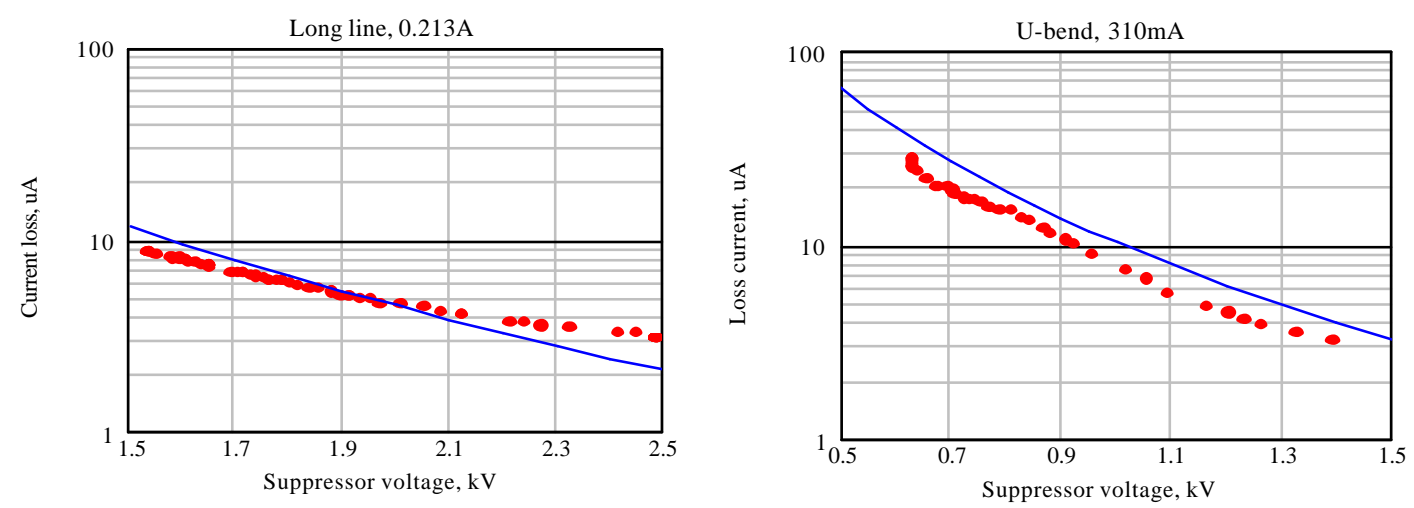

FIGURE 1. Measured and calculated current loss in long line (0.213A) and U-bend (0.310A)

\section{CONCLUSIONS}

1. Single IBS places a limitation for the minimum collector voltage $U_{\text {coll }}$. Acceptable level of losses of $\Delta=2.5 \cdot 10^{-6}$ corresponds to the minimum value of $U_{\text {coll }}=2 \mathrm{kV}$.

2. The r.m.s. energy spread of the beam due to the multiple IBS is below the acceptable limit for the electron cooling process.

3. Tails of the multiple IBS Gaussian distribution are insignificant in comparison with the single large-angle IBS tails.

4. Any increase of the beam size is beneficial for both the multiple and single IBS as it leads to a lower transverse temperature and lower beam density.

5. Loss dependence on the envelope seems to be weak in the long line and very strong in the U-bend.

6. Geometrical beam parameters are obtained using 4D formalism for coupled optics.

The authors are thankful to Valeri Lebedev (FNAL) for fruitful discussions and to Denis Artamonov (NSU, Novosibirsk, Russia) for his valuable help in the data measurements.

\section{REFERENCES}

1. S. Nagaitsev et al., "Antiproton cooling in the Fermilab Recycler", these Proc. (COOL05, Galena, 2005).

2. A. Burov, Ya. Derbenev, S. Nagaitsev and A. Shemyakin, "Optical principles of beam transport for relativistic electron cooling", Phys. Rev. ST-AB 3, p. 094002 (2000).

3. E. M. Lifshitz and L.P. Pitaevskii, "Physical Kinetics", Pergamon, 1981.

4. A. Burov, A. Shemyakin and S. Nagaitsev, FERMILAB-TM-2133 (2000).

5. A. Burov et al., "Optics of Electron Beam in the Recycler", these Proc.

6. N. Dikansky et al., Ultimate Possibilities of Electron Cooling, BINP Preprint 88-61 (1988).

7. A. Shemyakin et al., "Attainment of a high-quality electron beam for Fermilab cooler", these Proc.

8. V. Lebedev and A. Boga cz, "Betatron Motion with Coupling", e-print JLAB-ACC-99-19 (2001).

9. V. Lebedev, "OptiM", at http://www-bdnew.fnal.gov/pbar/organizationalchart/lebedev/OptiM/optim.htm 


\section{APPENDIX: PRINCIPAL AXES OF THE BEAM DISTRIBUTION}

Geometrical beam parameters can be calculated via generalized Twiss functions for coupled optics ${ }^{8}$. All functions for the simulation were calculated by OptiM code ${ }^{9}$. Particle coordinate vector can be represented through the linear combination of two complex vectors $\hat{\mathbf{v}}_{\mathbf{1}}(s)$ and $\hat{\mathbf{v}}_{2}(s)$ satisfying the equation $\mathbf{M}\left(s \mid s^{\prime}\right) \hat{\mathbf{v}}_{\mathbf{i}}(s)=e^{i \mu\left(s \mid s^{\prime}\right)} \hat{\mathbf{v}}_{\mathbf{i}}\left(s^{\prime}\right)$.

$$
\begin{gathered}
\hat{\mathbf{x}}(s)=\operatorname{Re}\left(\sqrt{2 I_{1}} e^{-i \psi_{1}} \hat{\mathbf{v}}_{\mathbf{1}}(s)+\sqrt{2 I_{2}} e^{-i \psi_{2}} \hat{\mathbf{v}}_{\mathbf{2}}(s)\right) \\
\hat{\mathbf{x}}=\left(\begin{array}{l}
x(s) \\
x^{\prime}(s)-\frac{e B_{s}}{2 p c} y(s) \\
y(s) \\
y^{\prime}(s)+\frac{e B_{s}}{2 p c} x(s)
\end{array}\right), \hat{\mathbf{v}}_{\mathbf{1}}=\left(\begin{array}{l}
\sqrt{\beta_{1 x}(s)} \\
-\frac{i(1-u(s))+\alpha_{1 x}(s)}{\sqrt{\beta_{1 x}(s)}} \\
\sqrt{\beta_{1 y}(s)} e^{i v_{1}(s)} \\
-\frac{i u(s)+\alpha_{1 y}(s)}{\sqrt{\beta_{1 y}(s)}} e^{i v_{1}(s)}
\end{array}\right), \hat{\mathbf{v}}_{\mathbf{1}}=\left(\begin{array}{l}
\sqrt{\beta_{2 x}(s)} e^{i v_{2}(s)} \\
-\frac{i u(s)+\alpha_{2 x}(s)}{\sqrt{\beta_{2 x}(s)}} e^{i v 2(s)} \\
\sqrt{\beta_{2 y}(s)} \\
-\frac{i(1-u(s))+\alpha_{2 y}(s)}{\sqrt{\beta_{2 y}(s)}}
\end{array}\right)
\end{gathered}
$$

$B_{s}$ is longitudinal magnetic field, $I_{i}, ?_{\mathrm{i}}$ are new canonical variables, actions and phases for the two transverse modes. Defining emittances (un-normalized) as $e_{1}=2 I_{1 \max }, e_{2}=\left\langle I_{2}\right\rangle$, the $4 \mathrm{D}$ emittance $\varepsilon_{T}=r_{c} \sqrt{m T_{c}} / p$ is a product of two emittances defined above ${ }^{8}: \varepsilon_{T}^{2}=\varepsilon_{1} \varepsilon_{2}$ with

$$
\varepsilon_{1}=\frac{B_{c} r_{c}^{2}}{B \rho}, \varepsilon_{2}=\frac{m c T_{c}}{p e B_{c}}, \frac{\varepsilon_{1}}{\varepsilon_{2}}=\frac{B_{c}^{2} r_{c}^{2}}{T_{c}} \frac{e^{2}}{m c^{2}} \approx 8 \cdot 10^{3},
$$

$B ?=p c / e=16.2 \mathrm{kG} \cdot \mathrm{cm}$ for the $4.35 \mathrm{MeV}$ electrons. Emittance $e_{1}$ corresponds to the total magnetic flux on the cathode and is responsible for the beam hydrodynamic motion and its shape. Emittance $e_{2}$ relates to the thermal velocities, driving intrabeam scattering. For the hydrodynamic mode, $x=\sqrt{2 I_{1} \beta_{1 x}} \cos \psi_{1}$ and $y=\sqrt{2 I_{1} \beta_{1 y}} \cos \left(v_{1}-\psi_{1}\right)$, yielding the half-axes $a_{?, ?}$, the beam cross-section $S$, and the axes tilt angle $\varphi_{1}$ :

$$
\begin{aligned}
& \left.a_{\xi, \eta}^{2}=I_{1}\left[\left(\beta_{1 x}+\beta_{1 y}\right) \pm \sqrt{\beta_{1 x}^{2}+2 \beta_{1 x} \beta_{1 y} \cos 2 \nu_{1}+\beta_{1 y}^{2}}\right)\right] \\
& S=\pi \varepsilon_{1} \sqrt{\beta_{1 x} \beta_{1 y}}\left|\sin \nu_{1}\right|, \quad \operatorname{tg} 2 \varphi_{1}=\frac{2 \sqrt{\beta_{1 x} \beta_{1 y}} \cos \nu_{1}}{\beta_{1 y}-\beta_{1 x}} .
\end{aligned}
$$

Emittance of the thermal mode $\varepsilon_{2}$ determines the r.m.s. transverse velocities in the beam frame in $\varphi_{2}$-tilted principal axes

$$
\begin{gathered}
\overline{v_{\tilde{x}, \tilde{y}}^{2}}=\frac{B \rho}{B_{c} \lambda_{\tilde{x}, \tilde{y}}} \frac{T_{c}}{m c^{2}}, \lambda_{\tilde{x}, \tilde{y}}=\frac{1}{2}\left[\left(\beta_{2 x}+\beta_{2 y}\right) \pm \sqrt{\beta_{2 x}^{2}+2 \beta_{2 x} \beta_{2 y} \cos 2 v_{2}+\beta_{2 y}^{2}}\right) \\
\operatorname{tg} 2 \varphi_{2}=\frac{2 \sqrt{\beta_{2 x} \beta_{2 y}} \cos v_{2}}{\beta_{2 y}-\beta_{2 x}} .
\end{gathered}
$$

